\title{
INDEX*
}

Emilija Grubačić

\section{KNICANIN / BANAT}

S. 7

Werner H. Veith

BOCKWITZ KR. SPROTTAU

S. 95

" Dieser dritte Monographienband enthält Einzelarbeiten aus den Gebieten 9 und 5 (s. a. Monumenta Germaniae Acustica, PHONAI 4, 1965, S. $245 \mathrm{ff}$. und PHONAI 8, S. $223 \mathrm{ff}$.). deren Tonbandaufnahmen ihrer Mundart nach zum Banater Rheinfränkischen und zu den schlesischen Diphthongierungsmundarten gehören. 
